\title{
Anaesthesia In Austere Environments
}

\author{
AJ Mellor
}

\section{Introduction}

This review will examine the possibilities for providing anaesthesia for casualties in challenging situations. There is a limited evidence base on which to draw as most anaesthetists faced with such problems will generate their own solution based on experience and personal preference. Military personnel are perhaps more likely to encounter these challenges and may be trained anaesthetists or junior doctors with limited anaesthetic experience. The review will highlight the principal problems of planning to provide anaesthesia in an austere environment and focus on the Triservice anaesthetic apparatus (the equipment trained anaesthetists are most likely to use in the field) and ketamine (arguably the best choice for a non anaesthetic trained doctor in a difficult environment).

\section{The Environment}

Boulton described four broad types of "difficult environment"(1);

Planned but isolated situations - in the military this could include the provision of anaesthesia on deployment or at sea or on an expedition. Provision of care is limited by personnel constraints, portability of equipment, re-supply etc.

- Extreme urgency - for example a casualty trapped at the scene of an accident due to a collapsed building.

- Disaster or battle conditions - where a team may be well equipped but re-supply or sudden changes in casualty numbers may overwhelm the organisation.

- Difficult environments within hospitals for example an MRI scanner.

The fourth scenario is beyond the scope of this review. The other three situations may apply equally to anaesthetists and non-anaesthetists.

This review cannot hope to cover all the

Surg Cdr AJ Mellor RN

Cardiothoriacic

Anaesthetist

Cardio Outpatients, James Cook University

Hospital, Marton Road, Middlesbrough,

TS3 4BW prises the speciality of anaesthesia. However, the information contained should guide the thought processes to enable any physician to plan providing the best possible anaesthetic solution given their background and specific skills.

\section{Principal Considerations}

An "austere" environment can be a relatively well provided for situation with logistic support, for example the field hospital, or the very different environment of a single handed medic on an expedition to a remote corner of the globe. There are some broad similarities in planning anaesthetic provision in either of these locations.

\section{Manpower constraints}

It is reasonable to assume that patients cared for in an austere environment will be trauma patients. Their care requires careful attention to fluid resuscitation pre-operatively (as discussed below), consideration to intra-operative care, post operative care and monitoring. Potentially all these tasks could fall to one medical officer. It is important at an early stage to identify the limits to which one can reasonably provide care. This is equally true in a situation with overwhelming casualties despite seemingly adequate medical provision.

\section{Equipment constraints}

This is less of an issue in the planned isolated situation that may occur in a field hospital, however is a major constraint for the expedition MO. Even the best planned and stocked field hospital may come under pressure when overwhelmed with casualties and prevented from re-supply due to local military activity. Prior planning is essential. For example the author elected not to take intubation equipment on a long expedition to the Himalayas, to be able to make use of a laryngoscope and endotracheal tube drugs, suction apparatus, a means of ventilating and a means of monitoring the patient were required. It was considered that this was too great a burden logistically and a compromise was reached whereby ketamine and kit for a surgical airway was carried. Many trauma patients may require repeated procedures e.g. debridement, dressing changes which will have logistic implications. The likely time to evacuation of the casualty will therefore have a major impact on planning.

\section{Patient considerations}


then a catastrophic fall in blood pressure will occur as induction reduces the sympathetic nervous system tone. This is likely to be more marked in the military population of young, fit individuals (26). The choice of anaesthetic agent will influence cardiovascular stability. Trauma patients, especially troops going into battle, are likely to have eaten shortly before injury and major trauma and pain will cause an ileus and delay gastric emptying. For these reasons all trauma patients are at risk of aspiration of gastric contents which adds considerable risk to general anaesthesia. Laryngeal reflexes can be preserved by using local anaesthesia but where general anaesthesia is essential steps should be taken to protect the airway. This can be achieved by pressure on the cricoid cartilage to occlude the oesphagus and placing a cuffed tube in the trachea. This is a manoeuvre requiring skill and the use of both anaesthetic agents and neuromuscular blocking drugs. One of the major advantages of the anaesthetic ketamine is that a state of dissociative anaesthesia can be induced with preservation of laryngeal reflexes (discussed below). Environmental factors may also create difficulties for patient care. Anaesthetised patients cannot regulate their own temperatures and so rapidly become hypothermic or hyperthermic depending on ambient temperature. Additionally, the location of the patient may create considerable difficulties, for example an anatomically normal airway may become very difficult to manage when the casualty is trapped in a motor vehicle and access is difficult.

\section{Inhalational Anaesthesia}

In UK hospitals most anaesthetics are maintained via the delivery of vapourised anaesthetics into the breathing system. In austere environments inhalational anaesthesia presents problems because of the equipment required to deliver a known quantity of vapour and the supply and availability of medical gases.

The Triservice Apparatus has now stood the test of time as the best known and well studied apparatus for providing inhalational anaesthesia from a portable system with requirement for little or no compressed gas supply (Figure 1). The system comprises of a self inflating bag and a vapouriser with the addition of a ventilator for providing positive pressure ventilation(2). Volatile anaesthetic agents are delivered via the Oxford Minature Vapouriser (OMV). This vapouriser holds 50 mls of agent and is temperature stabilized by means of a fluid reservoir in the base. The vapoursier has a three legged stand and can be tilted through 60 deg without spillage of agent.

A graduated scale is available for the agents halothane, and isoflurane which calibrate well in normal use. There is limited evidence that the apparatus performs well with sevoflurane (3) but that sufficiently high vapour concentrations may not be able to be delivered to achieve an inhalational induction (4). The output of the OMV can be affected by environmental conditions. At increased temperatures the output will be higher than the graduated scale whilst the converse is true of low temperatures. High gas flows through the apparatus due to the changes in patient's minute volume will reduce the output compared to the scale markings. Use of the sytem in pushover mode (ie ventilator pushing positive pressure tidal volume through the OMV) leads to a higher than expected output (5). The barometric pressure also has an effect. Because the saturated vapour pressure of the agent remains unchanged with altitude but barometric pressure falls, the vapour will constitute a greater proportion of the delivered gas mix for a given vapouriser setting. The situation becomes more complex as the anaesthetic effect is dependant on mass of agent delivered. Mass equals concentration multiplied by density and the density falls as barometric pressure falls. The result of this is that a higher concentration is delivered than the set concentration but the mass of agent is unchanged, therefore the OMV can be used at the calibrated settings for the desired anaesthetic effect (1).

The feature that has made the TSA so useful in austere environments and why it is still used as the apparatus of choice in British Military Field Hospitals, is that anaesthesia can be provided using air as the carrier gas. This is a major benefit in terms of the supply

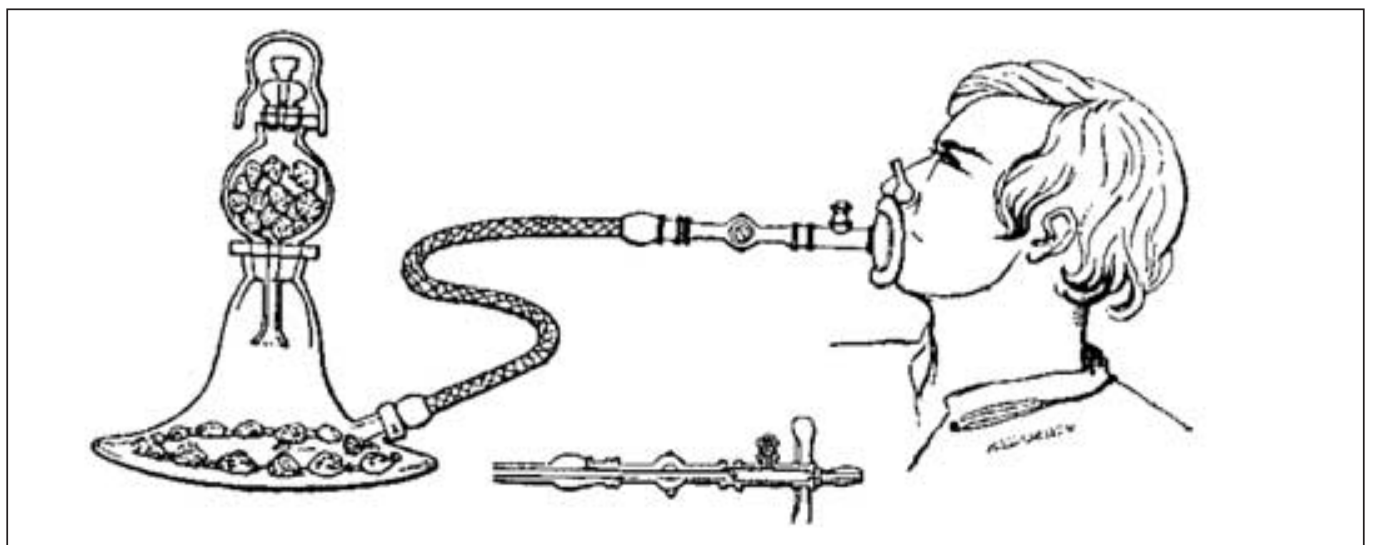

Fig 1. Diagram of Mr Hooper's ether inhaler. Reproduced from the Lancet 1847. 
and storage of compressed gases and also ensures that hypoxic gas mixtures cannot be delivered. In practice, where possible, the TSA is used with some supplemental inspired oxygen. From Houghton's initial work with the system the addition of 1 litre per minute of oxygen into the systems reservoir tubing provided an inspired oxygen concentration of $35 \%$ whereas the addition of $41 / \mathrm{min}$ provided an inspired oxygen concentration of $55 \%$ (at a minute volume of 8 litres)(2). Supplemental oxygen is required at $0.51 / \mathrm{min}$ to prevent hypoxaemia in spontaneously breathing subjects whilst positive pressure ventilation with air is adequate to prevent hypoxia (6).

\section{Intravenous Anaesthesia}

A number of agents have been available for use intravenously (IV), however in modern anaesthetic practise the list compromises propofol, thiopentone and ketamine.

\section{Thiopentone}

This drug was first made available as an IV agent in 1941. It was used to induce anaesthesia for several casualties after the Japanese bombing of Pearl Harbour and went down in anaesthetic folk lore as having caused more deaths than the Japanese! As is often the case the truth was not nearly so dramatic (7). Thiopentone is a sulphonated barbiturate, presented as a powder and reconstituted to a $2.5 \%$ solution. The usual induction dose of thiopentone is $2-4 \mathrm{mg} / \mathrm{kg}$ and has a duration of action of 15 mins. Repeat bolus doses can be given. The main drawback of thiopentone in the emergency situation is that it is a pronounced myocardial and respiratory depressant, an effect which is magnified in shocked patients (as at Pearl Harbour). A study of a small number of cases (11) of thiopentone (as sole agent) anaesthesia performed by an untrained anaesthetist resulted in apnoea in $27 \%$ of cases and one cardiac arrest (8). Thiopentone cannot be recommended for a non-anaesthetist in a difficult environment.

\section{Propofol}

Launched in 1986 propofol rapidly became the induction agent of choice in the UK. As experience of its use has grown it is now used for all types of procedures in all patient groups. Propofol is an oil at room temperature and insoluble in aqueous solution. Present formulations consist of $1 \%$ or $2 \%$ (w/v) propofol, $10 \%$ soyabean oil, $2.25 \%$ glycerol, and $1.2 \%$ egg phosphatide. An IV induction dose of $2 \mathrm{mg} / \mathrm{Kg}$ will be effective in one arm brain circulation time and will last for 5 mins. Propofol has a smaller initial volume of distribution than thiopentone and recovery from anaesthesia occurs after a period of redistribution. For this reason repeat doses can be given without any clinical indication of accumulation and prolonged effect (at least in the short term). Intravenous administration of the drug brings about anaesthesia, frequently apnoea and a drop in systemic blood pressure due to vasodilatation.

The only absolute contraindication to propofol is allergy which is very rare (it is formulated in egg phosphatide but appears to be safe to use in egg allergic patients).

Great care should be taken with propofol in the hypovolaemic patient due to changes, at least in part, in brain sensitivity to the drug (9). Shafer recommends that a patient in haemorrhagic shock should receive only 10$20 \%$ of the dose required in the healthy and even those patients who have been aggressively resuscitated require a $50 \%$ dose reduction. Shafer goes on to state that propofol is a "particularly poor choice for induction in patients with shock even after fluid resuscitation", it could be argued that the knowledge of enhanced effects of the drug in shock states allow its use to be titrated appropriately and safely. It should be noted that the half time for the plasma-effect site equilibration for hypnosis in patients is much shorter than that for systolic blood pressure (and times for systolic blood pressure increase with age). The clinical effect of this is that the patient will fall asleep before the full effects of the drug on blood pressure are seen (10).

It has been recommended that propofol mixed with alfentanil (a short acting synthetic opioid) provides a very useful combination for field anaesthesia (11). Propofol mixed with 2500 microgrammes of alfentanil in a $50 \mathrm{ml}$ syringe can be delivered by one syringe driver and requires no other anaesthetic equipment. The authors found that with this drug concentration the infusion could be set to run at a rate in mls equal to the patients body weight for the first 60 mins then reduced to run at weight/ 1.5 between $60-90$ mins before being reduced to weight/ 2 after that. By using this simple regimen satisfactory anaesthesia was maintained and emergence occurred as quickly as with inhalational agents.

Propofol is a very versatile and useful anaesthetic agent and has a place in planned isolated situations, however, in unskilled hands and without recourse to sophisticated anaesthetic equipment it should be avoided.

\section{Ketamine}

First described in 1965 the phencyclidine derivative ketamine has been very widely used in remote and difficult environments and is probably the closest agent to an ideal sedative agent. The drug is presented in ampoules of $10 \mathrm{mg}$ or $100 \mathrm{mg} / \mathrm{ml}$ and can be delivered via the IV, intramuscular(IM), nasal, oral or rectal routes of administration.

Ketamine induces a state of dissociative anaesthesia where patients appear to be awake and have little higher cortical depression yet the cortical awareness to external stimuli appears to be blocked. As brainstem activity remains normal the cardiac and respiratory centres remain largely unaffected. Profound 
analgesia occurs at the same time. Actions of the drug on the respiratory system include bronchodilation due to increases in circulating catecholamines and preservation of laryngeal reflexes. There is an often quoted increase in risk of laryngospasm following ketamine aneasthesia, however, this would not seem to be supported by the literature (12). Hodges used ketamine for sedation in children undergoing cleft palate surgery in Uganda in 130 patients with no respiratory complications (13). Blood pressure (BP) and cardiac output are increased with rises of systolic BP of $25 \%$ being reported (14). This is clearly beneficial in the trauma patient and is unique amongst induction agents, however, in patients with end stage shock with maximal circulating catecholamine levels this response is not seen and the drug is myocardial depressant. One very interesting effect of ketamine is that in animal models it has been demonstrated to have a marked neuroprotective effect mediated by antagonism of N-methyl-D-aspartate (NMDA) channels. Historically ketamine was considered to be contra-indicated in head injured patients due to its supposed effect of increasing intracranial pressure. Two recent studies have failed to find this effect and the evidence now points towards ketamine being at least safe (if not beneficial) in head injuries $(15,16)$. Interestingly from a military perspective NMDA receptors are involved in the central effects of organophosphorous compounds which may make ketamine the induction agent of choice in patients exposed to nerve agents(17). The drug is also safe to use at altitude with reports of it's use up to $3,900 \mathrm{~m}$ (18). Ketamine has a very wide therapeutic range with the median lethal dose (LD500 in animals being 100 times the human IV dose and 20 times the IM dose). One of the main reasons that ketamine use in the $\mathrm{UK}$ is low is the high incidence of emergence phenomena. This is said to occur because external stimuli are misinterpreted as a result of the dissociation effect. Emergence phenomena can, therefore, be kept to a minimum if the patient is allowed to recover in a quiet darkened area, in one study reassurance alone prevented any problems (19), however other authors have reported a high incidence requiring treatment with sedatives(8). Various sedative drugs (midazolam, haloperidol, lorazepam) have been given to reduce this side effect.

\section{Practical use of ketamine}

As with any anaesthetic agent the most reliable way to use the drug is with intravenous titration to the desired effect. Doses of less than $1 \mathrm{mg} / \mathrm{Kg}$ IV produce a significant amount of analgesia e.g. for realigning fractures, changing dressings. Larger doses produce the typical state of dissociation with glazed eyes and nystagmus. The maximum safe intravenous dose is $3 \mathrm{mg} / \mathrm{Kg}$. One technique involves placing $500 \mathrm{mg}$ of the drug in a bag of $500 \mathrm{ml}$ of dextrose, the drip is initiated (via a standard $15 \mathrm{drop} / \mathrm{ml}$ non-micro chamber drip) at $2 \mathrm{drops} / \mathrm{Kg} / \mathrm{min}$ until a satisfactorily deep level of anaesthesia is obtained (usually takes 2 minutes) after this the drip rate is reduced to $1 \mathrm{drop} / \mathrm{Kg} / \mathrm{min}$. The patient will wake approximately $10 \mathrm{mins}$ after the drip is stopped (20). A more sophisticated method was described by Restall and colleagues using $200 \mathrm{mg}$ of ketamine, midazolam (5mg) and vecuroinum (a neuromuscular blocking drug) $(12 \mathrm{mg})$ in a $50 \mathrm{ml}$ syringe of normal saline. By setting the infusion pump at $50 \%$ of the body weight (in mls) $2 \mathrm{mg} / \mathrm{Kg} / \mathrm{hr}$ of ketamine was delivered (21). Slow intravenous infusion is preferable to a rapid single bolus as occasional apnoea is seen with the drug. Intramuscular administration for anaesthesia should commence with a bolus dose of $4 \mathrm{mg} / \mathrm{Kg}$ (usual dose range $4-10 \mathrm{mg} / \mathrm{Kg}$ depending on depth of anaesthesia required). For analgesia $1 \mathrm{mg} / \mathrm{Kg}$ is adequate. Onset of action usually occurs within 5 minutes and lasts for approximately 30 minutes. Corresponding doses for other, more usual routes are $6-10 \mathrm{mg} / \mathrm{Kg}$ orally, 6$10 \mathrm{mg} / \mathrm{Kg}$ rectally and $6 \mathrm{mg} / \mathrm{Kg}$ nasally.

\section{Regional anaesthesia}

The use of local anaesthetics to provide analgesia when in difficult environments is immensely appealing. There is little evidence in the literature as to the use of local anaesthesia in austere environments although it is recommended in several reviews and anecdotal information available on the internet (1, 22). The United States military have recently introduced a training programme for anaesthetists to gain skills in regional anaesthesia and put these into practise in the developing world. It seems likely that with the increased use of combat body armour future conflicts will create numbers of casualties with predominantly extremity trauma. These cases will be particularly well suited to treatment with regional anaesthetic blocks. A recent study by the US military has shown that local blocks performed by anaesthetists with the patient then transferred to another operating area are acceptable to both patients and surgeons (23). This is clearly very advantageous for a hard pressed field hospital. Additionally patients treated with local anaesthesia will have less need for highly skilled staff, oxygen, analgesia and antiemetics in the recovery phase. The techniques of regional anaesthesia are too varied and specialized to be discussed in detail here, there are many standard texts on the subject $(24,25)$. Techniques of use may include axillary brachial plexus block for upper limb surgery, femoral block for femoral fractures and combined with sciatic blocks for lower limb surgery, lumbar and thoracic paravertebral blocks for surgery on the thorax and abdomen. These blocks can be performed relatively easily with a limited amount of training, however, gaining that experience (even for anaesthetic trainees) requires a dedicated approach - as in the US Army's regional 
anaesthesia initiative (22).

Simpler techniques are of use. The Advanced Trauma Life Support manual recommends the use of haematoma blocks to relocate fractured limbs (26). Local infiltration of a local anaesthetic, especially if continued (with patience) by the surgeon and combined with ketamine sedation can be very useful $(8,13)$. It is worth noting that prilocaine is an equally effective local anaesthetic agent to lignocaine and is less toxic allowing a dose of $400 \mathrm{mg}$ to be used in adult patients $(40 \mathrm{mls}$ of a $1 \%$ solution or up to $80 \mathrm{mls}$ if diluted to $0.5 \%)$.

Unfortunately for the expeditionary doctor most anaesthetic training in the UK provides training in neuroaxial (spinal or epidural) blocks to a greater extent than peripheral nerve blockade. Spinal anaesthesia has been reported in remote areas with no anaesthetist (8) with satisfactory results. In this paper, 32 patients underwent spinal blockade with a high incidence of hypotension (59.4\%) and a $12.5 \%$ incidence of headache (using a $21 \mathrm{G}$ needle). Head down tilt and intravenous fluid loading rapidly corrected the hypotension observed. Clearly issues of asepsis and the availability of vasoconstricitors, IV fluids etc make this a technique of use in the planned but isolated situation rather than in a disaster.

\section{Summary}

The scope of this review is by necessity somewhat limited and, as said in the introduction, methods used will depend on the skills and experience of the practitioner involved. The triservice apparatus remains the system of choice for delivering volatile anaesthetic agents to patients in areas without the infrastructure to supply an endless quantity of medical gases, the planned isolated or battle conditions of Boultons scenarios. The TSA's portability, robust design and versatility have stood the test of time. By reviewing intravenous agents the author has attempted to find an evidence base for the most appropriate agent for a non anaesthetic trained doctor finding his or herself called on to provide emergency anaesthesia in the field. The most useful drug is ketamine which has the advantage of cardiovascular stability, preservation of airway reflexes, ease of administration via different routes and versatility for providing analgesia, sedation or general anaesthesia.

\section{References}

1. Boulton TB. Anaesthesia and Resuscitation in Difficult Environments. Int Anesthesiol Clin 1973; 11:143-238.

2. Houghton IT. The Triservice Anaesthetic Apparatus. Anaesthesia 1981;36(12):1094-108.
3. EH Liu, SS Dhara. Sevoflurane Output from the Oxford Minature Vapouriser in Drawover Mode. Anaesthesia Intensive Care 2000;28:532-536 from our ref.

4. Mellor A, Hicks I. Sevoflurane delivery via the Triservice Apparatus. Anaesthesia In press.

5. Bell G, Ralph J. The Drawbacks of Pushovers. Anaesthesia 2000;55:1000-2.

6. Tighe SQ, Turner GA, Merrill SB, Pethybridge RJ Minimum oxygen requirements during anaesthesia with the Triservice anaesthetic apparatus. A study of drawover anaesthesia in the young adult. Anaesthesia 1991;46(1):52-6.

7. Bennetts FE. Thiopentone Anesthesia at Pearl Harbour. Br F Anaesth 1995;75:366-368.

8. Adenunkanmi AR. Where there is no anaesthetist: A study of 282 consecutive patients using intravenous, spinal and local infiltration anaesthetic techniques. Trop Doct 1999;29(2):56-7.

9. Shafer SL. Shock Values. Anesthesiology 2004; 101(3):567-568

10. Sneyd JR, Recent advances in intravenous anaesthesia. Br J Anaesth 2004;93(5):725-36.

11. Saissey J-M. Simplified use of mixed propofol and alfentanil for anesthesia in remote locations. Military Medicine 2000;165(3):195-200.

12. Green SM, Rothrock SG, Lynch EL: Intramuscular ketamine for pediatric sedation in the emergency department: safety profile in 1,022 cases. Ann Emerg Med 1998;31(6):688-97.

13. Hodges SC, Hodges AM. A protocol for safe anaesthesia for cleft lip and palate surgery in developing countries. Anaesthesia 2000;55:436-441.

14. Tweed WA, Minuck M, Mymin D: Circulatory responses to ketamine anesthesia. Anesthesiology 1972;37(6):613-9.

15. Bourgoin A, Albanese J, Wereszczynski N, Charbit M, Vialet R, Martin C. Safety of sedation with ketamine in severely head injured patients; comparison with sufentanil. Crit Care Med 2003;31:711-7.

16. HijaziY, Bodonian C, Bolon M, Salord F, Boulieu R. Pharmacokinetics and haemodynamics of ketamine in intensive care patients with brain or spinal cord injury. Br f Anaesth 2003:90:155-60.

17. Mion G, Tournier J-P, Petitjeans F, Dorandeu F, Lallement G, Ruttimann M. Neuroprotective and antiepileptic properties of ketamine in nerve agent poisoning. Anesthesiology 2003;98:1517.

18. Bishop RA, Litch JA, Stanton JM. Ketamine at high altitude. High Alt Med Biol 2000;1(2):111-4.

19. Sklar GS, Zukin SR, Reilly TA: Adverse reactions to ketamine anaesthesia. Abolition by a psychological technique. Anaesthesia 1981;36(2):183-7.

20. Li J, Ketamine: Emergency applications. E medicine. 27 Jan 2005; available from: http://www.emedicine. com/emerg/topic802.htm

21. Restall J, Tully AM, Ward PJ, Kidd AG. Total intravenous anaesthesia for military surgery. A technique using ketamine, midazolam and vecuronium. Anaesthesia 1988;43(1):46-9.

22. Breckenmeier CC, Lee EH, Shields CH, Sampson JB, Chiles JH. Regional Anesthesia in Austere Environments. Regional Anesthesia and Pain Medicine. 2003;28(4):321-7.

23. Waters JH, Leivers D, Maher D, Scanlon T, DeGuzman GM. Anesth Analg. 1997;84(4):773-6.

24. Jankovic D. Regional nerve blocks and infiltration therapy. Textbook and colour atlas. Blackwell Science. 2004.

25. Rosenberg PH (Ed). Local and regional anaesthesia. London: BMJ Books. 2000.

26. American College of Surgeons Committee on Trauma. Advanced trauma life support for doctors, student course manual. 7th ed. United States of America. 2004 